units, in the UK and Ireland. This represented the whole population. Data were analysed using SPSS, with open-ended questions grouped and themed.

Results A response rate of $65 \%$ revealed that $61.4 \%$ of respondents provided some level of lymphoedema service. Where services existed, $69.2 \%$ of hospices had staff employed solely to treat lymphoedema, $66.7 \%$ had ring-fenced budgets for lymphoedema care with $67.3 \%$ treating lymphoedema patients from outside the palliative population. Nurses and physiotherapists were the main professional groups involved in lymphoedema management.

MLD was employed as a treatment strategy for palliative patients in $75.6 \%$ of hospices. It was used holistically, often in an adapted form, with both physical and psychological benefit.

Discussion Wide geographical variations exist with a cluster of fully developed services in the Midlands. It appears that different models of care are evolving in Wales and Northern Ireland in response to recent national guidance.

Conclusions There is potential inequity of service provision due to geographical variations. Further research is needed to plot the trend of hospice lymphoedema services, explore the impact of new models of care and give further consideration to the use of MLD with palliative patients.

\section{P89 A REVIEW OF LYMPHOEDEMA SERVICE PROVISION IN ADULT HOSPICES IN THE UK AND IRELAND}

Kathy Birch, ${ }^{1}$ Wendy Knibb² ${ }^{1}$ Princess Alice Hospice, Esher, England; '2University of Surrey, Guildford, England

10.1136/bmjspcare-2011-000105.89

Introduction This study scoped lymphoedema services in adult hospices in the UK and Ireland. It was undertaken to benchmark and inform development of a local hospice lymphoedema service and was completed as part of an MSc in Advanced Practice.

Aims The study investigated hospice demographics, lymphoedema services including budget provision, treatments used, staffing levels and qualifications. A secondary aim explored the use of Manual Lymphatic Drainage (MLD) with the palliative population.

Methods The study was undertaken in November and December 2010. It adopted a quantitative approach using a postal questionnaire sent to 195 adult hospices, with in-patient 